DOI: $10.3901 / J M E .2019 .14 .215$

\title{
考虑人误的人机干涉排队网络模型研究
}

\author{
孔繁森 ${ }^{1,2}$ 白小刚 ${ }^{1,2}$ 李丛双 ${ }^{1,2}$ 陆俊点 ${ }^{1,2}$
}

(1. 吉林大学机械与航空航天工程学院 长春 130022;

2. 吉林大学未来工厂的设计与规划吉林省重点实验室 长春 130022)

\begin{abstract}
摘要: 以单元制造系统作为研究背景, 探讨一人多机情况下单元制造系统性能的评价问题。根据实际机加单元工况, 将操作 工人在操作过程中存在的两类失误分别定义为可修复和不可修复性人误, 并建立人误排队网络模块。在此基础上建立考虑人 误的零件/机器开环排队网络模型和机器/工人闭环排队网络模型, 描述考虑人误时的人机干涉机制; 并以此为基础给出了考 虑人误的单元制造系统人机干涉性能评价计算流程; 利用提出的模型和方法对一个实际工程案例进行了仿真研究, 研究结果 表明: 当考虑人误时, 人机比变化对系统人机干涉影响最为显著, 而人机作业比越大, 系统对人误的承受能力就越低, 即上 料/下料操作失误会导致人机干涉水平增强, 也会增加操作人员的作业负荷。
\end{abstract}

关键词: 人机干涉; 排队网络模型; 人员失误; 人机比; 人机作业负荷比

中图分类号: TB18

\section{Research of Queuing Network Modelling of Human-machine Interference by Considering Human Error}

\author{
KONG Fansen $^{1,2}$ BAI Xiaogang ${ }^{1,2}$ LI Congshuang ${ }^{1,2}$ LU Junrui ${ }^{1,2}$
}

(1. School of Mechanical and Aerospace Engineering, Jilin University, Changchun 130022;

2. Jilin Provincial Key Laboratory of Designing and Planning for Factory, Jilin University, Changchun 130022)

\begin{abstract}
Based on the research background of cell manufacturing system, the performance evaluation of cell manufacturing system under the condition of one person and multiple machines is discussed. According to the actual working conditions of the machining unit, two kinds of errors in the operation process of the operator are defined as repairable and irreparable human errors respectively, and the human errors queuing network module is established. Then an open-loop queuing network model considering human errors and a closed-loop queuing network model of machine/operator are established to describe the human-machine interference mechanism considering human errors. On this basis, a calculation flow chart for evaluating the human-machine interference performance of cellular manufacturing system considering human errors is given. A practical engineering case is simulated by using the proposed model and method. The results show that when human error is considered, the change of human-machine ratio has the most significant impact on human-machine interference. The larger the proportion of human-machine operation, the lower the system's tolerance to human error. That is to say, the presence of feeding/cutting errors will lead to the enhancement of human-machine system interference level and increase the workload of operators.
\end{abstract}

Key words: human-machine interference; queuing network model; human error; man-machine ratio; man-machine load ratio

\section{0 前言}

\section{人机干涉在一人多机的精益生产系统中普遍存} 在，调查表明，在一人多机制造系统中，机器会浪 费超过 $10 \%$ 的时间在人机干涉上，显然，若不能对 人机干涉现象进行有效控制便会造成生产资源的浪

* 国家科技重大专项(2017ZX04017001，2016ZX04004)和吉林省科技发展计 划(2017010117JC)资助项目。20180725 收到初稿, 20190105 收到修改稿
费。文献对人机干涉系统的研究主要集中在人机比 和人员服务规则两个方向上。所使用的研究方法和 技术手段主要有绘制图表法、数学模型法、排队论 法、排队网络模型法和计算机仿真分析法等。其中, 陈建严 ${ }^{[1]}$ 通过绘制人机作业表的方法描述了人员服 务作业量和机器服务周期, 对比差异后获得人员相 对机器的利用率和人机干涉时间。HADAD 等 ${ }^{[2-3]}$ 使用一个多项式模型描述了一种人机干涉问题，他 们所提出的模型不必考虑机器故障与服务时间分布 
问题。NGUYEN 等 ${ }^{[4]}$ 提出一种利用信息熵法, 以最 小成本作为目标确定最优人机比的方法。JOHN 等 ${ }^{[5]}$ 则专门探讨了考虑机器故障分布的人机干涉问题。 NIEBEL 等 ${ }^{[6]}$ 为了获得最优的人机比, 通过仿真发 现人员服务机器的数量和人机干涉时间两个参数相 关, 并通过与传统静态方法的对比指出如何使用仿 真来克服利用静态人机图获得人机比的缺陷。 KHINTCHINE $^{[7]}$ 采用 GI/G/1 模型分析了闭环柔性装 配系统问题, 并指出在处理干涉问题时, 两阶矩法 可以获得良好的近似精度。ELSAYED 等 ${ }^{[8]}$ 针对制 造单元中的机器人与加工机床之间的干涉问题, 以 成本最小为目标探讨了机器人与机器之间的最佳组 合。KELLY ${ }^{[9]}$ 通过对零件和机器之间以及机器和操 作员之间的交互作用建模, 分别以开环和闭环网络 的形式解决了单个操作员、工作单元机器干扰问题。 在工作单元里, 机器零件必须按顺序经过。

蒋悦 ${ }^{[10]}$ 建立一种自调节功能的排队网络模型 去解决人机干涉问题。使用该模型可验证人力配置 与设备能力的关系。ELICANO ${ }^{[11]}$ 为美国中西部某大 型制造工厂建立了一个仿真模型，使用该模型可以 模拟人机数量的搭配方案, 并满足人和机器的停机 成本最小这一目标。宋明华 ${ }^{[12]}$ 建立了由多台不同机 器组成的仿真模型, 该模型可以模拟加工速率和服 务需求不同时的人机干涉状态。DESRUELLE 等 ${ }^{[13]}$ 给出一种快速确定工作单元产能在现有配置和运作 方法下是否能够满足客户需求的方法, 作者将工作 单元简化为两个相互影响的排队网络进行分析, 其 中一个排队网络是开环的工件/机器排队网络, 另一 个是闭环的机器/工人排队网络。YANG 等 ${ }^{[14]}$ 在 DESRUELLE 的模型基础上考虑了机器故障对系统 产能的影响。

前述研究表明, 在影响人机干涉系统性能的众 多因素中, 前人的研究多集中在可控因素如人机比 对系统性能的影响, 关心的是机器利用率, 人员利 用率, 以及不可控因素如机器故障对人机干涉系统 性能的影响, 很少考虑不可控因素人误对人机干涉 行为的影响, 尽管人误可控, 但人误是很难避免的, 显然对该问题的研究具有重要的理论意义和实际价 值。本文采用排队网络模型和数学建模相结合的方 法研究了考虑人误的人机干涉问题。

\section{1 考虑人误的人机干涉排队网络模型}

本文将人误划分为两类，可修复性与不可修复 性人误。可修复性人误是指工人上料或者下料时出 现的失误, 具体失误及修复过程如图 1 所示, 本文
假定当工人出现这种失误时, 工人一次重新作业即 可修复失误。可修复失误延长了上料和下料的时间， 增加了操作工人的劳动负荷, 降低了生产效率。

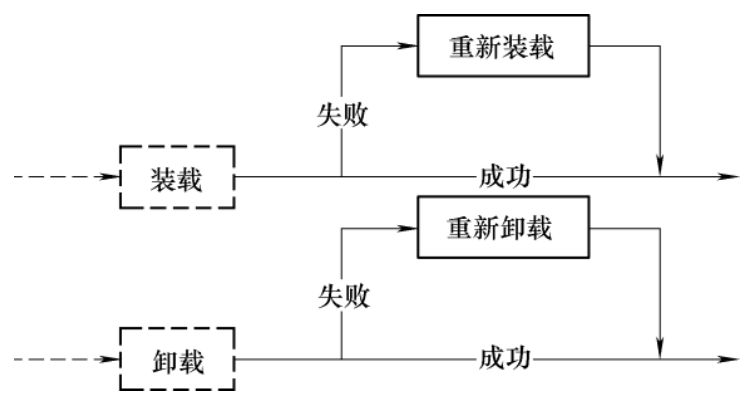

图 1 可修复人误网络模型

不可修复失误是指在工人对机器设备进行调整 作业时出现的失误，这种失误是工人本身在生产前 无法发现，并会产生废品。考虑到在调整阶段出现 失误会导致机器加工出来的零件变为废品, 因此, 这种失误浪费了机器的正常加工时间, 削减了机器 的生产能力。这种不可修复的失误导致机器的加工 时间增长, 为描述这个增长量, 作者在有效加工之 前加入一个加工延迟时间, 这种失误的影响机制如 图 2 所示。延迟时间等于机器无效加工时间, 延迟 时间过后所进行的作业为正常加工作业，不会再产 生废品。

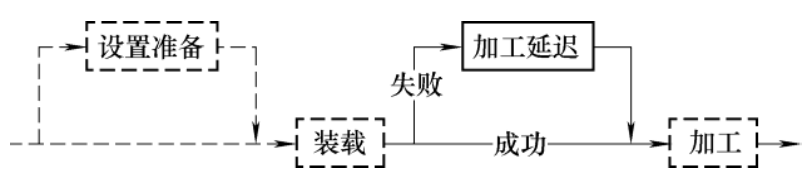

图 2 不可修复人误网络模型

本文将可修复和不可修复人误问题都转换为工 人作业时间的延迟问题，在此基础上建立考虑人误 的人机干涉排队网络模型。

在前述定义基础上本文假设如下。

（1）假设缓存区不会出现阻塞，但是会出现工 作站饥饿情况。

(2) 假设各个机器的故障停机概率为零。

(3) 工人在服务过程中的失误概率不为零。

(4) 工人在各机器之间的行走时间可忽略不计。

\section{1 零件/机器开环排队网络模型试验方法}

如图 3 所示, 在零件/机器排队网络模型中, 机 器是服务者, 零件是被服务对象, 并使用多个小开 环排队网络模型来模拟整个生产单元。由于假设缓 存区不会出现阻塞, 因此, 当系统达到稳态时, 工 作站的产出率等于工作站毛坏零件的到达率 $\lambda_{p}$, 零 件的到达率 $\lambda_{p}$ 按照客户的需求量设定, 但需要满足 零件流经各个工作站的平均耗时 $\tau$ 要小于零件的平 均到达间隔时间 $1 / \lambda_{p}$, 以避免出现系统阻塞情况。 


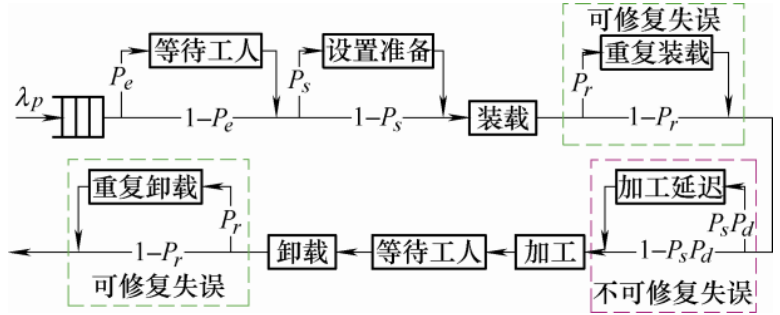

图 3 考虑人误的零件/机器开环排队网络模型

假设: 零件到达工作站, 工作站刚好处于空闲 等待状态的概率为 $P_{e}$, 此时, 零件停留在工作站内 等待操作工人前来处理。显然工作站处于繁忙状态 的概率为 $1-P_{e}$, 此时, 零件存储在缓存区内。

假设: 到达零件与前一个到达零件不属于同一 批次的概率为 $P_{s}$, 需要进行调整作业, 而不需要调 整的概率为 $1-P_{s}$;

假设: 发生上料失误的概率为 $P_{r}$, 则, 不发生 上料失误的概率就为 $1-P_{r}$;

如果需要调整作业, 且发生了调整作业的失误, 则出现加工延迟的概率为 $P_{s} P_{d}$ 。加工延迟后, 零件 自动进入加工状态, 加工结束后, 零件等待操作工 人下料。

假设: 工人发生下料失误的概率也为 $P_{r}$, 下料 失败后工人会及时采取措施, 失误修复的方式为重 新下料, 本文假定一次重新下料即可完成失误修复, 工人将完成卸载的零件转运到缓存区。

为了评价零件流经工作站的平均耗时 $\tau$, 需要 先计算出人机干涉时间 $(I)$, 干涉时间 $I$ 的求解需要 借助机器/工人闭环排队网络模型进行求解。

\section{2 机器/工人闭环排队网络模型}

机器/工人闭环排队网络模型如图 4 所示, 在排 队网络中, 操作工人为服务者, 数量为 1 , 机器为
被服务的对象, 数量为 $N$ 。网络分两个阶段: 第一 个阶段描述工人对机器进行操作服务, 此阶段工人 完成对机器的 “上料”、“下料” 和必要的 “调整” 作业。由图 3 可以看出, 每一台机器的零件到达率 为 $\lambda_{p}$, 那么在闭环排队网络中有 “上料” 和 “下料” 服务需求的机器到达率为 $N \lambda_{p}$, 有 “调整” 作业需 求的机器到达率为 $N \lambda_{p} P_{s}$ 。考虑到可修复性人误的存 在, 在 “上料” 和 “下料” 环节嵌入人的修复操作 模块。由于前述假设操作工人出现上料和下料的失 误概率均为 $P_{r}$, 所以流经 “重新上料” 和 “重新下 料” 环节的流量均为 $N \lambda_{p} P_{r}$; 第二阶段描述机器对 零件进行加工服务, 在稳态系统中, 假设零件到达 工作站时工作站空闲, 且工作站前缓存区为空的概 率为 $P_{e}$, 所以, 零件完成 “下料” 作业到达 “机器 等待零件” 作业的路径流量为 $N \lambda_{p} P_{e}$, 若有新零件 到达, 则刚完成 “下料” 作业的机器进行 “上料” 作业, 其路径流量为 $N \lambda_{p}\left(1-P_{e}\right)$ 。完成 “上料” 作业 的机器自动进入第二阶段完成 “加工” 作业, 或者 进入 “机器等待零件” 队列等待新零件到达。若操 作工人出现调整失误, 机器会经历一个 “加工延迟” 时间, 此时路径流量为 $N \lambda_{p} P_{s} P_{d}$, 若操作工人未出现 调整失误, 机器会在 “上料” 后直接进入 “加工” 队列, 路径流量为 $N \lambda_{p}\left(1-P_{s} P_{d}\right)$ 。当机器完成 “加工” 作业或者有新的零件到达空闲机器时, 机器从第二 阶段转移到第一阶段。由于一位操作工人服务 $N$ 台 机器, 所以两条路径汇总为一个 “机器等待服务” 队列, 汇总路径流量为 $N \lambda_{p}\left(l+P_{e}\right)$, 然后队列中机器 再进入第一阶段接受操作工人服务, 如此往复循环。 排队网络模型遵从流量守恒, 通过简单的加法、减 法运算即可求解出其他各路径流量。

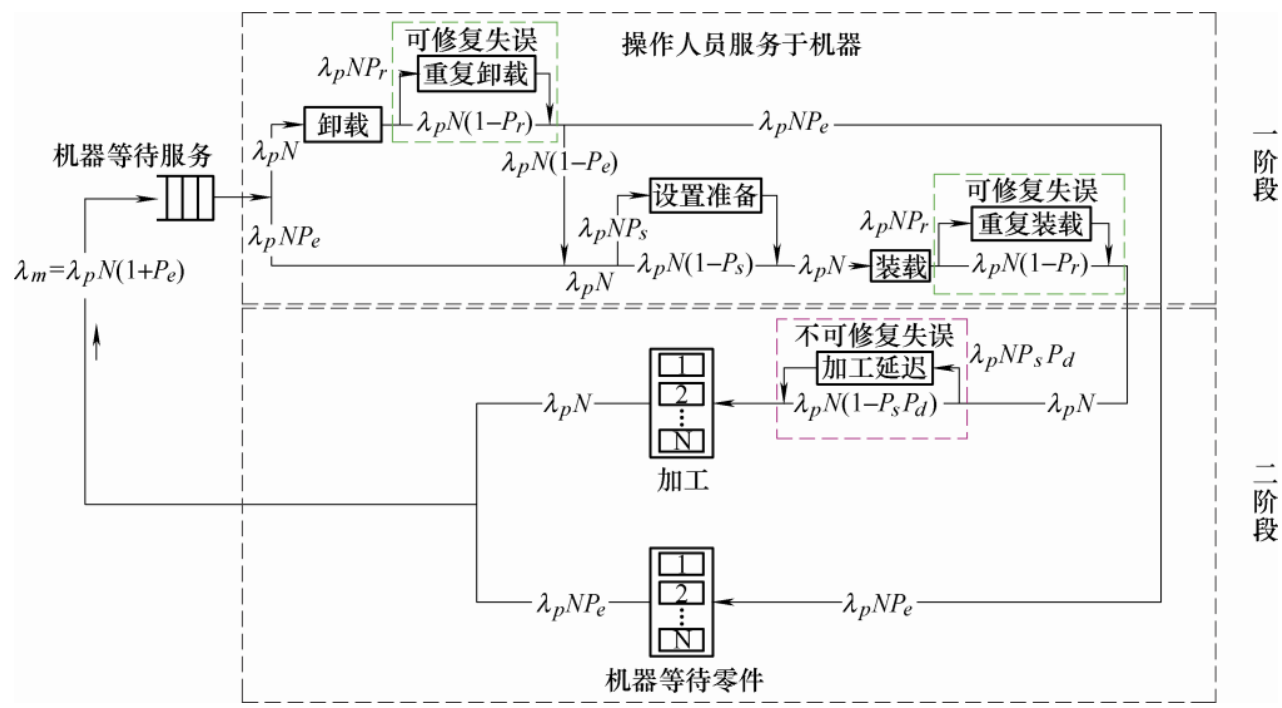

图 4 考虑人误的机器/工人闭环排队网络模型 
操作工人在服务机器时有 5 种可能的服务路径, 即, 下料作业、上料作业、调整作业 + 上料作业、下 料作业 + 上料作业和下料作业+调整作业 + 上料作业。

\section{2 考虑人误的人机干涉系统性能评价}

\section{1 工作站占用率}

工作站占用率是指生产一个产品平均占用工作 站的时间与零件到达时间间隔的比值, 用于描述工 作站在生产过程中的繁忙程度。

$$
\rho_{\text {sta }}=\lambda_{p} \tau
$$

式中, $\rho_{s t a}$ 为工作站占用率, $\tau$ 为零件流经由单人/ 单机构成的工作站用时，在零件到达间隔时间 $1 / \lambda_{p}$ 内, 工作站占用率为 $\rho_{\text {sta }}$ 。

$$
P_{e}=1-\lambda_{p} \tau
$$

式中, $P_{e}$ 为某一零件到达工作站时, 此工作站处于 空闲等待的概率。

由图 5 可知, 零件流经工作站的平均耗时包含 了零件等待工人时间、调整时间、零件的上/下料时 间(包括因上/下料失误而产生的重新上/下料时间)、 加工延迟时间、机器时间、机器等待时间。

$$
\begin{gathered}
\tau=P_{e} I+P_{s} E[S]+E[L]+P_{r} E\left[L_{r}\right]+P_{s} P_{d} E[D]+ \\
E[P]+I+E[U]+P_{r} E\left[U_{r}\right]
\end{gathered}
$$

式中, $\tau$ 为零件流经工作站平均耗时, 包括在缓存 区等待的时间。 $I$ 为干涉时间, $P_{e}$ 某一零件到达工 作站时发现此工作站处于空闲等待的概率, $I P_{e}$ 即零 件到达工作站时, 机器处于空闲等待工人服务的时 间; $P_{s}$ 为某一零件到达工作站时需要工人对机器进 行调整作业的概率, $E[S]$ 即零件批次切换时工人对 工作站进行调整作业所需时间的平均值, $P_{S} E[S]$ 即 零件到达时工人进行设置作业的平均时间; $L$ 工人 进行上料零件作业所需的时间, $E[L]$ 工人上料零件 作业所需的平均时间; $L_{r}$ 工人重新上料零件所需的 时间, $P_{r}$ 工人在进行上料或下料作业时，上料或下
料作业操作失误的概率, $P_{r} E\left[L_{r}\right]$ 因失误重新上料或 下料作业的平均时间; $D$ 工人对机器进行调整作业 时的失误操作而造成机器的加工延迟时间, $P_{d}$ 工人 在进行调整作业时, 操作失误的概率, $P_{s} P_{d} E[D]$ 准 备作业失误的平均时间； $U$ 工人进行下料零件作业 所需的时间, $E[U]$ 工人进行下料零件作业所需的平 均时间; $U_{r}$ 工人重新下料零件所需的时间, $P_{r} E\left[U_{r}\right]$ 工人重新下料零件所需的平均时间。整理式(2)和(3) 可得

$$
\begin{gathered}
\tau=\left(P_{e} I+P_{s} E[S]+E[L]+P_{r} E\left[L_{r}\right]+P_{s} P_{d} E[D]+\right. \\
\left.E[P]+E[U]+P_{r} E\left[U_{r}\right]\right) /\left(1+\lambda_{p} I\right) \\
P_{s}=\sum_{i=1}^{P T} \frac{C_{i}}{l_{i}} / \sum_{i=1}^{P T} C_{i}
\end{gathered}
$$

式中, $P T$ 为零件类型的数量, $C_{i}$ 为顾客对第 $i$ 种零 件的年需求量, $l_{i}$ 为零件 $i$ 的批量。又因为

$$
\begin{aligned}
L_{r} & =L \\
U_{r} & =U \\
d_{i} & =p_{i}
\end{aligned}
$$

式(6)、(7)分别表示重新上料(下料)的时间与上料(下 料)时间相同, 式(8)表示零件的设置作业延误时间等 于机器加工时间, $p_{i}$ 为 $i$ 类型零件的机器加工时间。 根据式(6) (8), 将式(4)转化为

$$
\begin{gathered}
\tau=\frac{2 I+P_{s} E[S]+\left(1+P_{r}\right) E[L]}{1+\lambda_{p} I}+\frac{\left(1+P_{s} P_{d}\right) E[P]}{1+\lambda_{p} I}+ \\
\frac{\left(1+P_{r}\right) E[U]}{1+\lambda_{p} I} \\
E[S]=\sum_{i=1}^{P T} \frac{C_{i} s_{i}}{l_{i}} / \sum_{i=1}^{P T} \frac{C_{i}}{l_{i}} \\
E[P]=\sum_{i=1}^{P T} \frac{C_{i} p_{i}}{l_{i}} / \sum_{i=1}^{P T} \frac{C_{i}}{l_{i}}
\end{gathered}
$$

式中, $s_{i}$ 为加工 $i$ 类型零件时, 工人对工作站进行调 整作业所需的时间。式(10)为 $E[S]$ 的算法, 式(11) 为 $E[P]$ 的算法。

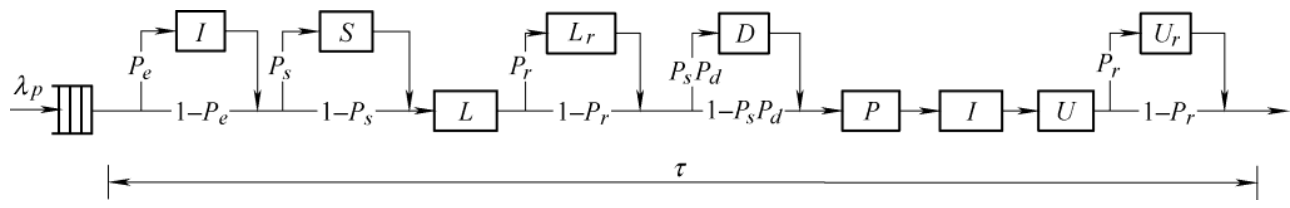

图 5 零件流经工作站耗时

显然, 如要获得工作站占用率 $\rho_{s t a}$ 和空闲率 $P_{e}$, 首先要给出干涉时间 $I$, 为此需要进一步对人和机 器的闭合排队网络模型进行分析。

\section{2 人员利用率}

人员利用率是工人的平均服务时间与机器到达
时间间隔的比值，用于描述的操作人员的繁忙程度。

$$
\rho_{o p}=\lambda_{m} E\left[S_{o p}\right]
$$

式中, $\rho_{o p}$ 为人员利用率, $\lambda_{m}$ 为机器到达工人服务队 列的到达率, $E\left[S_{o p}\right]$ 为操作工人服务时间的平均值。 
根据此前定义, 机器到达服务队列的到达率为

$$
\lambda_{m}=\lambda_{p} N\left(1+P_{e}\right)
$$

操作者的服务时间 $S_{o p}$ 是一个随机变量, 可以表达为

$$
S_{o p}=Z_{U}\left(U+P_{r} U_{r}\right)+Z_{L}\left(L+P_{r} L_{r}\right)+Z_{s} S
$$

整理后得

$$
S_{o p}=\left(1+P_{r}\right)\left(Z_{U} U+Z_{L} L\right)+Z_{s} S
$$

式中, $L 、 U$ 和 $S$ 三个参量分别表示工人完成零件上 料、下料和调整作业所需时间。 $Z_{L} 、 Z_{U}$ 和 $Z_{S}$ 在 $(0,1)$ 之间取值, 为 0 值代表没有进行该项作业, 取值为 1 代表进行该项作业。系统长时间运行, 上料和下 料作业发生的平均概率为 $E\left[Z_{L}\right]$ 和 $E\left[Z_{U}\right]$, 可表示为

$$
E\left[Z_{L}\right]=E\left[Z_{U}\right]=\frac{\lambda_{p} N}{\lambda_{m}}=\frac{1}{1+P_{e}}
$$

同样，调整作业发生的平均概率 $E\left[Z_{S}\right]$ 可以表示为

$$
E\left[Z_{S}\right]=\frac{\lambda_{p} N P_{s}}{\lambda_{m}}=\frac{P_{s}}{1+P_{e}}
$$

假定 $L 、 U$ 和 $S$ 彼此相互独立, 那么操作工人的平 均服务时间为

$$
E\left[S_{o p}\right]=\frac{\left(1+P_{r}\right)(U+L)}{1+P_{e}}+\frac{P_{s} E[S]}{1+P_{e}}
$$

根据式(12) (18) 得

$$
\rho_{o p}=\lambda_{m} E\left[S_{o p}\right]=\lambda_{p} N\left[\left(1+P_{r}\right)(U+L)+P_{s} E[S]\right]
$$

\section{3 机器有效利用率}

机器的 “有效利用率” 定义为在一个零件流经 工作站的总耗时 $\tau$ 内, 机器用于完成零件加工作业 的有效时间(包括机加工时间、上/下料时间和调整 时间)占比, 用 $\rho_{m}$ 表示, 则有

$$
\rho_{m}=\frac{E[P]+L+U+P_{s} E[S]}{\tau}
$$

机器的有效利用率可以非常灵敏地反映人误对 机器利用情况的影响。

\section{4 干涉时间}

首先将图 4 所示闭环排队网络分解为近似的两 阶段 $\mathrm{GI} / \mathrm{G} / 1$ 队列, 然后采用平均值分析法求解人机 干涉时间 $I$ 如下

$$
I=\mathrm{f}\left(E[W]_{\infty}\right) E[W]_{\infty}
$$

式中, $E[W]_{\infty}$ 为客户到达服从一般分布, 且待服 务客户为无穷多时, 客户的平均等待时间。 $c^{2}$ 为 某变量的平方变异系数(方差与平均值平方的比 值), 式(21)表示干涉时间是客户平均等待时间的 函数。

$$
E[W]_{\infty}=\frac{\left(c_{a}^{2}+c_{s}^{2}\right) E\left[S_{o p}\right] \rho_{o p}}{2\left(1-\rho_{o p}\right)}
$$

式中, $c_{a}^{2}$ 及 $c_{s}^{2}$ 的求解过程参照 KUEHN 研究 GI/G/1 队列时提出的迭代公式 ${ }^{[15]}$ 。

$$
\begin{gathered}
f\left(E[W]_{\infty}\right)=\frac{K(N-1)}{N} \times \frac{1}{1+\frac{E[W]_{\infty}}{E\left[S_{o p}\right]} \times\left[1-\frac{K(N-1)}{N}\right]} \\
K=\frac{Q_{N-1} / N}{Q_{N} / N} \\
Q_{N}=N \times \frac{E\left[S_{o p}\right] \times\left(1+Q_{N-1}\right)}{E\left[S_{o p}\right] \times\left(1+Q_{N-1}\right)+E[O]} \\
E[O]=\frac{1}{1+P_{e}}\left(E[P]+P_{s} P_{d} E[D]\right)+\frac{P_{e}}{\lambda_{p}\left(1+P_{e}\right)}
\end{gathered}
$$

根据式(21) (26), 可计算出干涉时间。式(23) (24)中 $K$ 为状态转移系数, $Q_{N}$ 为在排队网络中进入 $N$ 个客户时, 工作站前队列长度的期望值, $E[O]$ 为 客户在延迟工作站的滞留时间。根据上述原理, 本 文给出了考虑人误的单元制造系统人机干涉性能参 数(工作站占用率、人员利用率、机器有效利用率和 干涉时间)计算流程如图 6 所示。

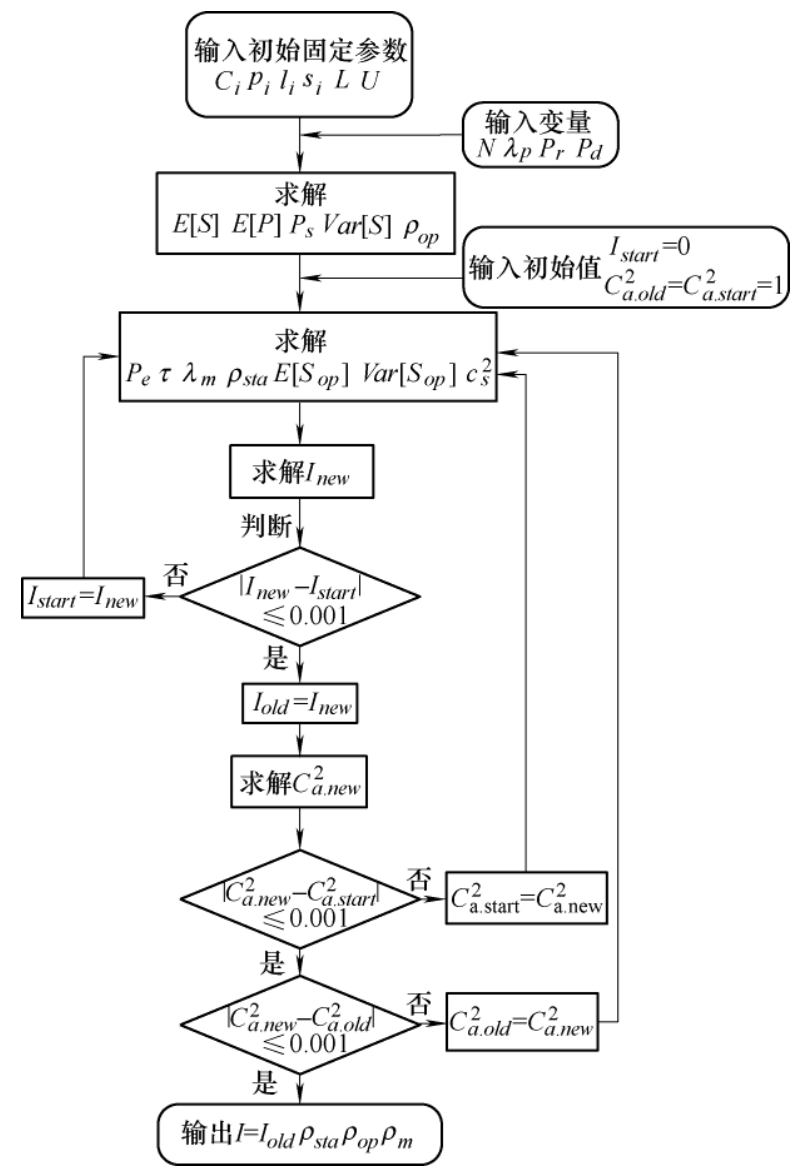

图 6 考虑人误的单元制造系统人机干涉性能评价流程图 


\section{5 人机作业比}

人机作业比是指在零件生产过程中工人操作时 间与机器加工时间的比值, 求解公式如下

$$
\beta=\frac{L+U+P_{s} E[S]}{E[P]}
$$

式中, $\beta$ 为人机作业比, 描述了零件生产过程中手 工作业所占的比例大小。工人操作时间包括上/下料 时间和调整时间, 总作业时间等于工人操作时间与 机加工时间之和。

\section{3 案例研究}

案例来源于文献[16], 背景为某公司机械加工 车间的太阳轮生产单元。如图 7 所示, 该单元由两 位工人和五台机器组成, 零件依次流经五台机器完 成全部加工。工人 1 服务前三台机器(车床、插齿 机 1 和插齿机 2), 完成前三道工序, 工人 2 服务后 两台机器(一台钻床和一台键槽插床), 完成后两道 工序。

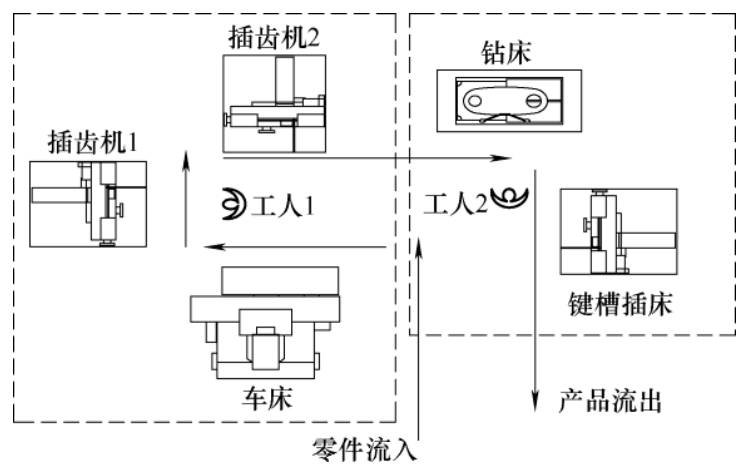

图 7 太阳轮生产单元
该生产单元负责生产五种型号的太阳轮, 假设 该生产单元是稳态的，即同种零件流经各工作站的 机加时间相同。其中主要生产参数如表 1 所示。

表 1 各类零件生产参数表

\begin{tabular}{cccccc}
\hline 零件类型 & $\begin{array}{c}\text { 需求量 } \\
\text { /件 }\end{array}$ & 批量 & 批次 & $\begin{array}{c}\text { 调整时间 } \\
\text { /min }\end{array}$ & $\begin{array}{c}\text { 机加时间 } \\
\text { /min }\end{array}$ \\
\hline 1 & 920 & 10 & 92 & 40 & 20 \\
2 & 600 & 10 & 60 & 40 & 20 \\
3 & 600 & 10 & 60 & 50 & 15 \\
4 & 840 & 10 & 84 & 35 & 15 \\
5 & 450 & 10 & 45 & 50 & 30 \\
\hline
\end{tabular}

经测时得到工人的上料时间 $L=3 \mathrm{~min}$, 下料时 间 $U=2 \mathrm{~min}$, 零件平均到达间隔时间为 $40 \mathrm{~min}$, 假 设第一类人误概率 $P_{r}=5 \%$, 第二类人误概率 $P_{d}=3 \%$, 到达率 $\lambda_{p}=0.25$ 。依据测时结果经统计分析得到 $E[S]=41.847, E[P]=19.208, P_{S}=0.1, \operatorname{Var}[S]=33$ 。

按照图 7 对太阳轮生产单元进行建模, 将该单 元拆分为一人服务三机排队网络系统(图 8)和一人 服务两机排队网络系统(图 9)。

如图 8 所示, 一人三机零件、机器和操作工人 联合排队网络模型由零件流通和机器流通两种路 径组成, 零件流通路径和机器流通路径都会经过 “加工” 队列, 代表零件要进入队列被机器加工, 同时也代表机器要进入队列完成对该零件的加工 作业。当两种路径汇聚到 “上料”、“重复上料”、 “下料”、“重复下料” 及 “调整” 环节时, 操作工 人对发出服务申请对象进行相应的服务, 实际上是 操作工人在服务机器的同时, 机器也在对零件进行 服务。

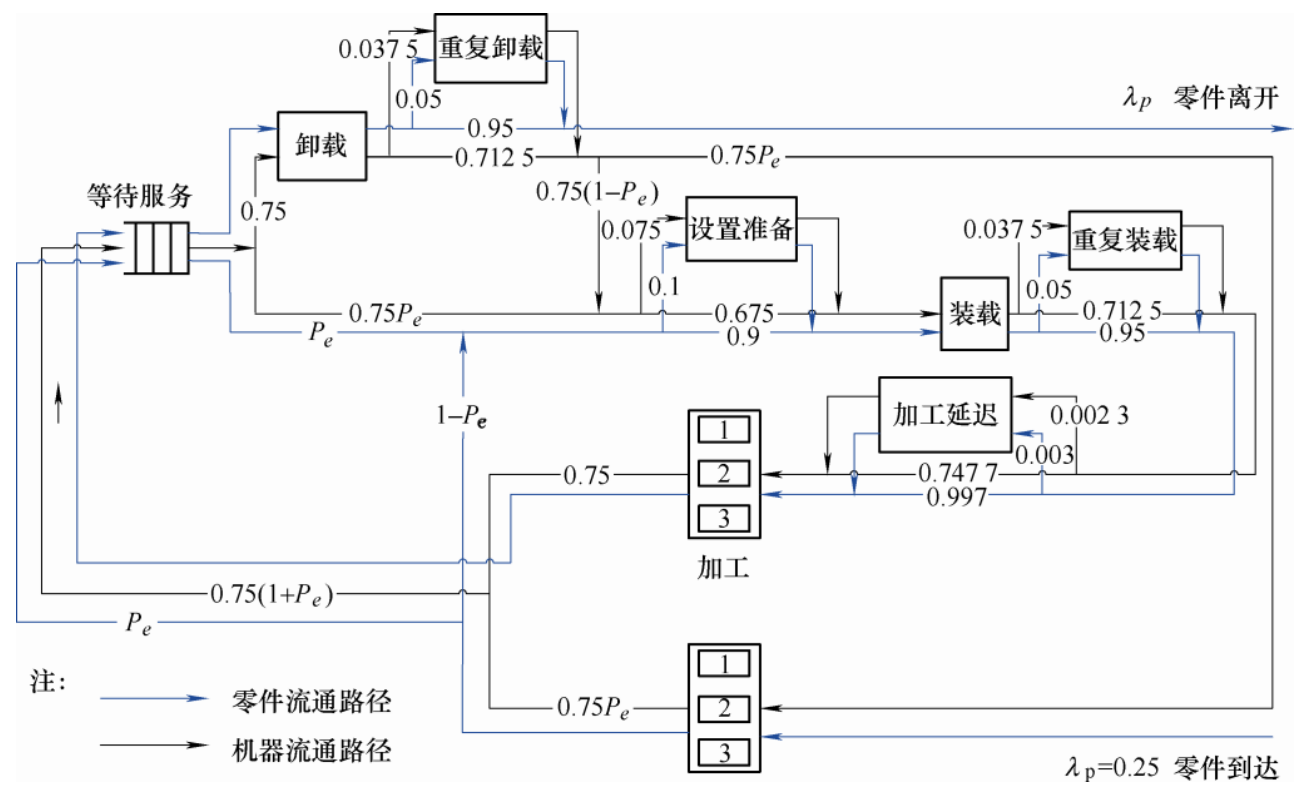

图 8 考虑人误的一人三机系统的联合排队网络模型 
整理工人 1 所在的一人服务三机系统, 迭代过 程的输入参数分别如表 2 所示。

表 2 一人服务三机系统程序输入参数表

\begin{tabular}{|c|c|c|c|c|c|c|c|c|c|}
\hline $\begin{array}{l}E[P] \\
/ \mathrm{min}\end{array}$ & $\begin{array}{l}E[S] \\
/ \mathrm{min} \\
\end{array}$ & $\begin{array}{c}L \\
/ \mathrm{min} \\
\end{array}$ & $\begin{array}{c}U \\
/ \mathrm{min} \\
\end{array}$ & $N$ & $\begin{array}{c}P_{r} \\
(\%) \\
\end{array}$ & $\begin{array}{c}P_{d} \\
(\%)\end{array}$ & $\begin{array}{c}P_{s} \\
(\%)\end{array}$ & $\begin{array}{l}\text { Var } \\
{[S]}\end{array}$ & $\lambda_{p}$ \\
\hline 19.208 & 41.847 & 3 & 2 & 3 & 5 & 3 & 10 & 33 & 0.25 \\
\hline
\end{tabular}

将以上参数输入到图 6 所示迭代程序中, 运行 程序, 输出结果如表 3 所示。

\section{表 3 一人服务三机系统程序输出参数表}

\begin{tabular}{cccc}
\hline $\begin{array}{c}\text { 干涉时间 } \\
I / \mathrm{min}\end{array}$ & $\begin{array}{c}\text { 工作站占用率 } \\
\rho_{s t a}(\%)\end{array}$ & $\begin{array}{c}\text { 人员利用率 } \\
\rho_{o p}(\%)\end{array}$ & $\begin{array}{c}\text { 机器有效利用 } \\
\text { 率 } \rho_{m}(\%)\end{array}$ \\
\hline 10.589 & 98.5 & 70.7 & 71.9 \\
\hline
\end{tabular}

从输出结果可以看出, 平均的机器等待工人时 间(干涉时间)已超过 $10 \mathrm{~min}$, 而机器的平均加工时 间 $E[P]=19.208 \mathrm{~min}$, 也就是机器每加工完一个工 件, 需要花费 $10 \mathrm{~min}$ 等待工人前来服务, 可见一人 服务三机的系统, 机器的效率非常低的, 此时工作 站的占用率 $\rho_{s t a}$ 已达到 $98.5 \%$, 这说明人机工作站几 乎已达到满负荷, 同时也表明零件流经工作站的耗 时较长, 而人员利用率 $\rho_{o p}$ 及机器有效利用率 $\rho_{m}$ 都 接近于 $70 \%$ ，还有一定的可提升空间。

图 9 为工人 2 所在生产单元考虑人误的一人 两机联合排队网络模型。该模型的输入参数如表 4 所示。

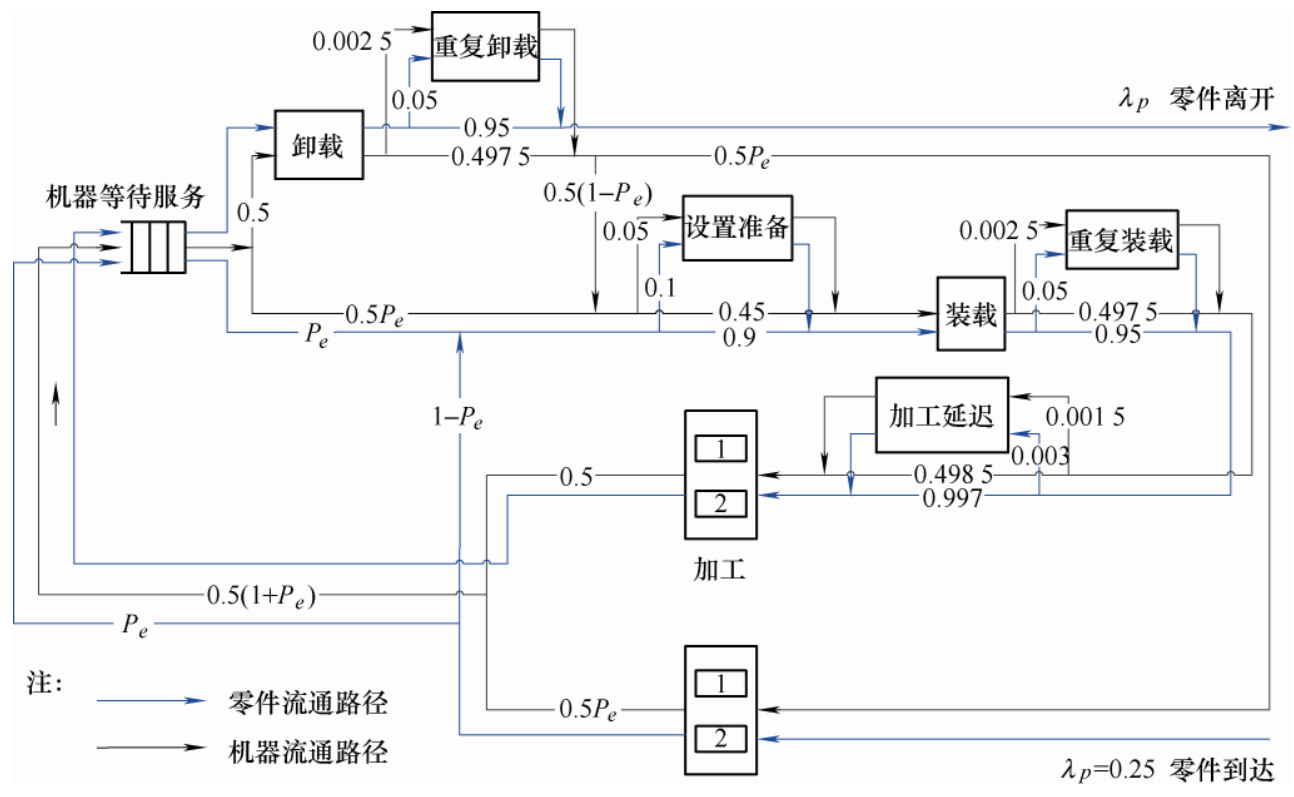

图 9 考虑人误的一人两机系统联合排队网络模型

表 4 一人服务两机系统程序输入参数表

\begin{tabular}{|c|c|c|c|c|c|c|c|c|c|}
\hline $\begin{array}{l}E[P] \\
/ \mathrm{min}\end{array}$ & $\begin{array}{l}E[S] \\
/ \mathrm{min}\end{array}$ & $\begin{array}{c}L \\
/ \mathrm{min}\end{array}$ & $\begin{array}{c}U \\
/ \mathrm{min}\end{array}$ & $N$ & $\begin{array}{c}P_{r} \\
(\%)\end{array}$ & $\begin{array}{c}P_{d} \\
(\%)\end{array}$ & $\begin{array}{c}P_{s} \\
(\%)\end{array}$ & $\begin{array}{l}\text { Var } \\
{[S]}\end{array}$ & $\lambda_{p}$ \\
\hline 19.208 & 41.847 & 3 & 2 & 2 & 5 & 3 & 10 & 33 & 0.25 \\
\hline
\end{tabular}

同样, 将以上参数输入到图 6 所示迭代程序中, 运行程序, 输出结果如表 5 所示。

表 5 一人服务两机系统程序输/出参数表

\begin{tabular}{cccc}
\hline $\begin{array}{c}\text { 干涉时间 } \\
I / \mathrm{min}\end{array}$ & $\begin{array}{c}\text { 工作站占用率 } \\
\rho_{s t a}(\%)\end{array}$ & $\begin{array}{c}\text { 人员利用率 } \\
\rho_{o p}(\%)\end{array}$ & $\begin{array}{c}\text { 机器有效利用率 } \\
\rho_{m}(\%)\end{array}$ \\
\hline 3.459 & 81.9 & 47.1 & 86.6 \\
\hline
\end{tabular}

对比表 3 和表 5 中的数据也可以发现, 与一人 服务三机的输出相比, 一人服务两机系统的各输出 参数都有较大的变化。其中, 干涉时间从一人服务 三机的 $10.589 \mathrm{~min}$ 降为 $3.459 \mathrm{~min}$, 下降了 $67.3 \%$,
工作站占用率 $\rho_{s t a}$ 从 $98.5 \%$ 下降到 $81.9 \%$, 人员利用 率从 $70.7 \%$ 下降到 $47.1 \%$, 机器有效利用率由原来 的 $71.9 \%$ 上升到 $86.6 \%$ 。

\section{4 人误对性能指标的影响}

\section{1 人误与人机比对生产系统性能影响}

为了研究人误和人机比对生产系统性能的共 同影响, 本文依据实际生产中的常用人机配置情 况, 分别讨论当 1 人服务 2 台、 3 台和 4 台机器 时, 人误率变化对人机系统性能的影响, 并假定 第一类人误率 $P_{r}$ 的取值区间为 $(0,30 \%)$, 取值间 隔为 $2 \%$, 第二类人误率 $P_{d}=5 \%$, 其它参数设置 同表 2, 经过 48 次迭代运算, 输出结果如图 10 13 所示。 


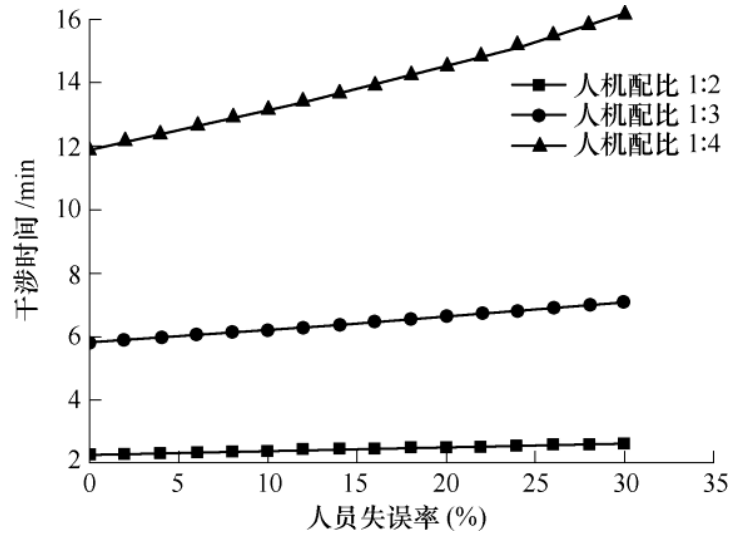

图 10 人机比及人员失误率对干涉时间的影响

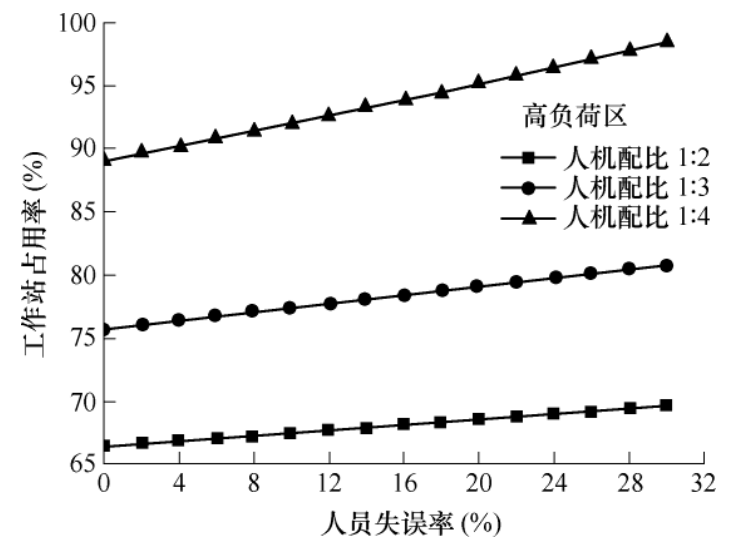

图 11 人机比及人员失误率对工作站占用率的影响

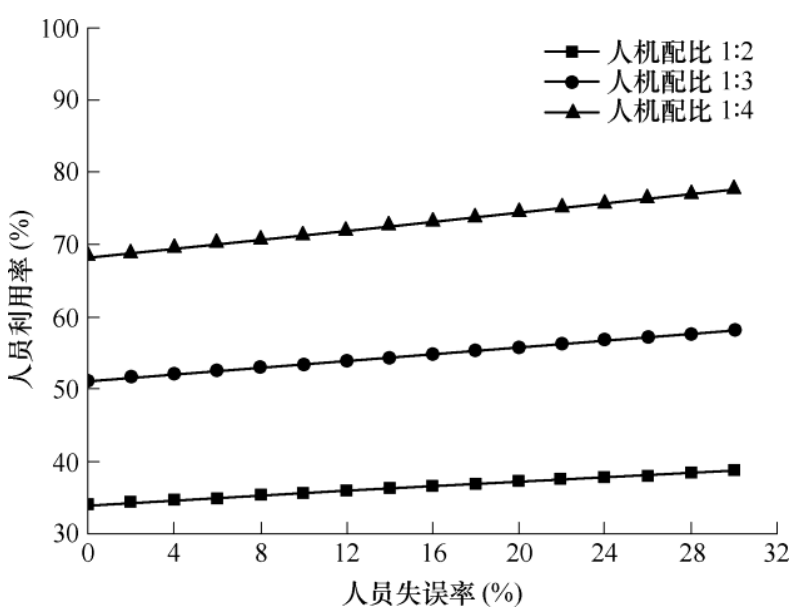

图 12 人机比及人员失误率对人员利用率影响

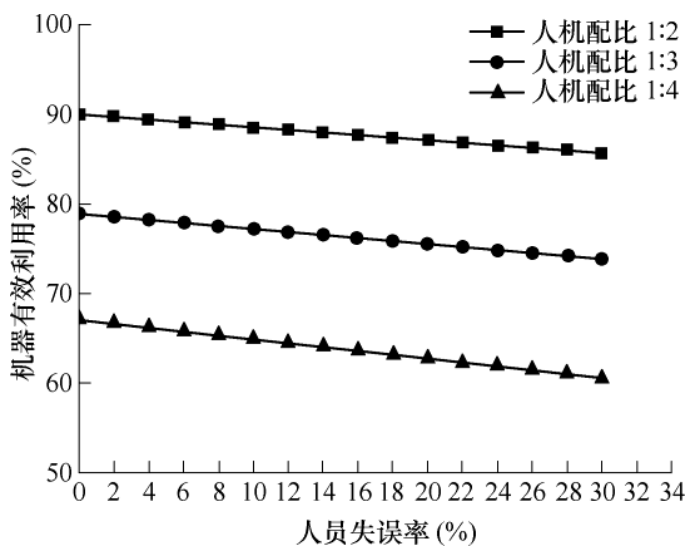

图 13 人机比及人员失误率对机器有效利用率的影响
对比三种人机比发现：伴随着工人服务机台数 的增加，系统的干涉时间、工作站占用率及人员利 用率都有上升, 而机器的有效利用率却在下降。各 性能指标与人误率呈近似线性关系, 且从图 10 可以 看出在任意人误率下，每增加一台机器干涉时间都 会成倍增长, 可见人机比是影响系统干涉水平的重 要因素。

由图 11 可见, 当人机比为 $1: 4$ 时, 人误率超 过 20\%后，工作站的占用率已高于 $95 \%$ ，工作站处 于高负荷状态。由图 12 可见: 人员利用率与人机比 及人员失误率之间呈现近似线性关系, 并且相邻两 条线之间的间距也相同，这说明人机比的差异不会 对人员失误率与人员利用率之间的关系产生影响, 这一结果与式(19)所表达的含义是一致的。

\section{2 人误与人机作业负荷比对系统性能的影响}

这里设第一类人员失误率 $P_{r}$ 的取值区间为 $(0,30 \%)$, 取值间隔为 $2 \%$ ，第二类人员失误率 $P_{d}=0$, 其他参数设置同表 2 , 选定三个水平的人 机作业负荷比 $(\beta=0.4 、 0.7 、 1)$, 研究人误率对系统 性能的影响。经 48 次运行迭代程序后得到结果如 图 14 17 所示。

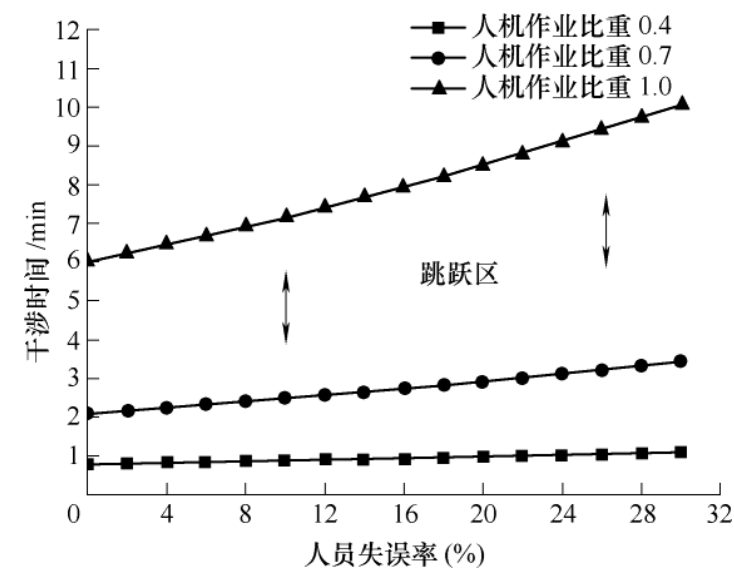

图 14 人机作业比重与人员失误率对干涉时间的影响

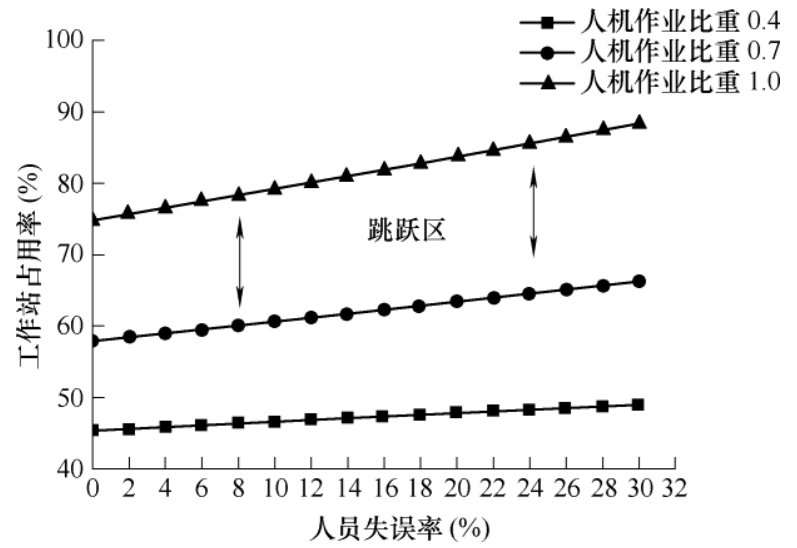

图 15 人机作业比重与人员失误对工作站占用率的影响 


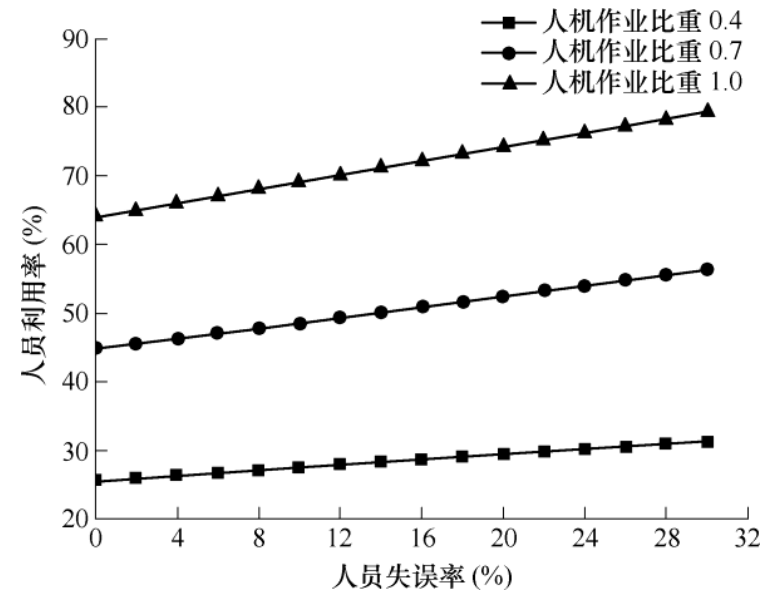

图 16 人机作业比重与人员失误对人员利用率的影响

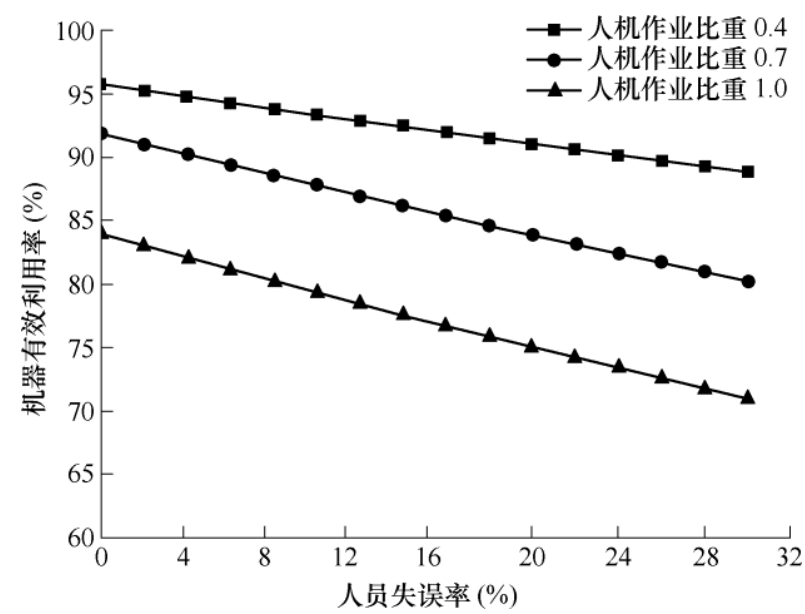

图 17 人机作业比重与人员失误对机器有效利用率的影响

由图 14 17 可见: 人机作业比重的变化对人误 率与各项性能指标之间的线性关系影响不大。当人 机作业比增加时, 干涉时间、工作站占用率及设备 有效利用率都有很明显波动, 在 $\beta=0.7$ 与 $\beta=1$ 两条 曲线之间有较大的 “跳跃” 区。当人机作业比接近 于 1 时, 人员失误率对系统的干涉水平、工作站占 用率、人员利用率的影响将更为显著。

由图 16 可以看出, 人机作业比重趋近于 1 时不 会产生影响曲线间的 “跳跃区”, 但随着人机作业比 的提升, 人误率变化对人员利用率的影响趋势愈加 显著。

\section{5 结论}

(1) 在一人多机系统中, 各系统指标与人误概 率呈近似线性关系, 并且人员服务的机台数越多, 干涉时间受人员失误的影响越显著, 因此, 在进行 制造系统设计时应优先考虑人机比的设定问题。

(2) 在一人多机系统中, 人机作业比重越大, 系统对人员失误的承受能力就越低, 如需避免这种 情况的发生, 可适当的将人员的作业任务向机器进
行转移。

（3）人员利用率与人机比及人员失误率之间呈 现近似线性关系，并且相邻两条线之间的间距也相 同，这说明人机比的差异不会对人员失误率与人员 利用率之间的关系产生影响。

本文研究的是考虑人误时的人机干涉问题, 那 么将这一问题弱化, 如何考虑由于工人存在能力差 异而对人机系统性能产生的影响问题需要进一步 研究。

\section{参 考 文 献}

[1] 陈建严. S 公司生产线的人机比与经济性研究[D]. 广 州: 华南理工大学, 2014.

CHEN Jianyan. Man-Machine matching and economic analysis research for S corporation[D]. Guangzhou: South China University of Technology, 2014.

[2] HADAD Y, KEREN B, GUREVICH G. A multinomial model for the machine interference problem with different service types and multiple operators[J]. Computers \& Industrial Engineering, 2013，66(2): 264-273.

[3] HADAD Y, KEREN B, GUREVICH G. Calculation of the steady state waiting time distribution in the context of the machine interference problem[J]. International Journal of Operational Research，2016， 26(4): 443-459.

[4] NGUYEN DM, TAI HAL, TAO PD. Solving the multidimensional assignment problem by a Cross-Entropy method[J]. Journal of Combinatorial Optimization, 2014, 27(4): 808-823.

[5] JOHN PALESANO, JEYA CHANDRA. A machine interference problem with multiple types of failures[J]. International Journal of Production Research, 1986, 24(3): 567-582.

[6] ANDRIS FREIVALDS, BENJAMIN NIEBEL. Niebel`s methods, standards and work design[M]. New York: McGraw Hill Education, 2013.

[7] KHINTCHINE A. On mean times of stoppage of machines[J]. Mathmaticeski Sbornic, 2009, 13 : 167-180.

[8] ELSAYED E A, NORTON J J. Machine interference in automated cells [J]. Annals of Operations Research, 1987, 9 (1): 449-468.

[9] KELLY F P. Networks of queues[J]. Advanced Applied Probability, 1998, 5(18): 744-756.

[10] 蒋悦. 制造系统人力配置优化建模与研究 $[D]$. 上海: 上 海交通大学, 2010.

JIANG Yue. Labor allocation optimization modelling [D]. Shanghai: Shanghai Jiao Tong University, 2010.

[11] ELICSNO R V. How many helpers in a pool[J]. American 
Machinist, 2007, 19(2): 291-305.

[12] 朱明华. 芯片制造企业生产现场人机比仿真分析及应 用研究[D]. 重庆: 重庆大学, 2012.

ZHU Minghua. The simulation analysis and applied research about MMR in chip manufacturing enterprise [D]. Chongqing: Chongqing University, 2012.

[13] DERUELLE P, STEUDELS H J. A queuing network model of a single-operator manufacturing work cell with machine/operator interference[J]. Management Science, 1996, 42(4): 576-590.

[14] YANG T, LEE R S, CHEN MC et al. Queueing network model for a single-operator machine interference problem with external operations[J]. European Journal of
Operational Research, 2005, 167(1): 163-178.

[15] KUEHN P J. Approximate analysis of queuing networks by decomposition[J]. IEEE Transactions on Communications, 1979, 27(1): 113-126.

[16] 李丛双. 单元制造系统中人机干涉问题研究[D].长春: 吉林大学, 2016

LI Congshuang. Research on human-machine interference problem in cell manufacturing system [D]. Changchun: Jilin University, 2016

作者简介: 孔繁森(通信作者), 男, 1965 年出生, 教授, 博士研究生导 师。主要研究方向为工业工程相关领域, 工厂设计与规划, 制造系统建 模仿真与优化，以人为核心的生产工程问题。

E-mail: kongfs@jlu.edu 